\title{
Combined Bifrontal Transcranial Direct Current Stimulation and Tailor-Made Notched Music Training in Chronic Tinnitus
}

\author{
Ho Yun Lee, Myoung Su Choi, Dong Sik Chang, and Chin-Saeng Cho \\ Department of Otorhinolaryngology, Eulji University Medical Center, Eulji University, Daejeon, Korea
}

Received October 20, 2016

Revised December 6, 2016

Accepted December 27, 2016

\author{
Address for correspondence \\ Ho Yun Lee, MD, PhD \\ Department of Otorhinolaryngology, \\ Eulji University Medical Center, \\ Eulji University, \\ 95 Dunsanseo-ro, Seo-gu, \\ Daejeon 35233, Korea \\ Tel $+82-42-611-3133$ \\ Fax +82-42-611-3136 \\ E-mail hoyun1004@gmail.com
}

Background and Objectives: We evaluated the short-term treatment outcomes of combined bifrontal transcranial direct current stimulation (tDCS) and tailor-made notched music training (TMNMT) in tinnitus patients. The associations of patient characteristics with treatment responsiveness were investigated. Subjects and Methods: Four sessions of bifrontal tDCS (F4: anode, F3: cathode) and TMNMT were conducted over a 2-week period in tinnitus patients. For tDCS, the stimulation intensity was $1.5 \mathrm{~mA}$ and the duration was approximately 20 min. During tDCS, patients listened to music lacking the frequency band within 1 octave of the tinnitus frequency. Patients were also instructed to listen to this music at home for at least 2 hours per day. One month after the final tDCS session, loudness (LD), awareness (AW), annoyance (AN), and effect on life (EL) of tinnitus were assessed subjectively using a visual analog scale. Results: A total of 14 patients were enrolled in this study. After treatment, a $50 \%$ or greater improvement in AN, AW, EL, and LD was observed in 57.1, $42.9,35.7$, and $28.6 \%$ of patients, respectively. Furthermore, $78.6 \%$ of patients showed a $50 \%$ or greater improvement in their tinnitus handicap inventory scores. For AN, the absence of sleep disturbance was significantly associated with treatment responsiveness ( $p=0.041, \mathrm{OR}=24.0$ ). Conclusions: Combined bifrontal tDCS and TMNMT is a promising treatment for chronic tinnitus. To maximize the treatment outcomes of this therapy, sleep disturbances should also be addressed in candidate patients.

J Audiol Otol 2017;21(1):22-27

KEY WORDS: Tinnitus · Neuromodulation · Prognosis · Treatment · Music.

\section{Introduction}

Tinnitus refers to the perception of sound in the absence of an external auditory stimulus. According to a recent report, $29.3 \%$ of the one nation population experiences tinnitus that interferes with daily life [1]. The suggested pathophysiologic mechanism of tinnitus includes maladaptive plastic changes in the brain due to the deafferentation of peripheral auditory input [2]. Thus, the phantom perception of sound is thought to be the manifestation of compensatory, persistent neural hyperactivity in the tonotopic cortical area [3].

Recently, a diverse array of neuromodulation techniques believed to alter cortical excitability has been introduced for

This is an Open Access article distributed under the terms of the Creative Commons Attribution Non-Commercial License (http://creativecommons.org/licenses/by-nc/4.0/) which permits unrestricted non-commercial use, distribution, and reproduction in any medium, provided the original work is properly cited. the control of neuropsychiatric disorders such as depression, pain, epilepsy, and chronic tinnitus [2]. These techniques most famously include transcranial direct current stimulation (tDCS). tDCS is designated to deliver a weak direct current of $0.5-2 \mathrm{~mA}$ to the scalp using surface electrodes, and is thought to induce changes in cortical excitability by shifting the resting membrane potential of affected neurons. In addition, lasting after-effects of tDCS are associated with the modulation of gamma-aminobutyric acid-ergic (GABAergic) and glutamatergic synapses [4]. According to polarity, anodal tDCS results in an increase in cortical excitability by depolarizing neurons and cathodal tDCS decreases excitability by hyperpolarization [2,5]. The most frequently used settings for tDCS stimulation intensity, duration, and frequency are $1.0-2.0 \mathrm{~mA}$ for $10-30$ minutes over $5-6$ sessions, although these parameters differ between studies [2,3,5-7].

The main stimulation sites for tDCS include the prefrontal 
cortex (PFC), auditory cortex (AC), and left temporoparietal area (LTA). Bifrontal tDCS of the bilateral dorsolateral PFC (DLPFC) is one of the most commonly used methods and has merit for modulating tinnitus-related anxiety and depression in a manner that can be customized to treat accompanying neuropsychiatric symptoms [6]. Indeed, different functional connectivity and resting state activity around the right $\mathrm{AC}$ and parahippocampus have been reported between responders and non-responders following tDCS [8].

Tailor-made notched music training (TMNMT) is another novel treatment strategy for tinnitus that aims to reduce cortical activity by enhancing lateral inhibition. At the initiation of TMNMT, an audiologist measures the individual patient's tinnitus frequency and allows the patient to listen to a filtered version of his or her favorite music that lacks the frequency band within an octave width of the tinnitus frequency. Significant decreases in the loudness of tinnitus have been reported following 12 months of regular TMNMT [9].

A recent study combining tDCS of the left AC with TMNMT reported that the polarity of tDCS (anodal or cathodal stimulation) did not affect treatment outcome; however, contradictory outcome results were reported via different questionnaires within patients [10]. Furthermore, to our knowledge, no report to date has studied the effect of combined bifrontal tDCS and TMNMT on tinnitus. Therefore, we conducted a prospective study of 14 tinnitus patients to evaluate the short-term treatment outcomes of bifrontal tDCS combined with TMNMT, and to determine the factors predicting treatment responsiveness.

\section{Subjects and Methods}

\section{Data collection and ethical statement}

Between July and December of 2015, we enrolled patient volunteers who visited the Eulji University Medical Center complaining of unilateral, non-pulsatile, subjective tinnitus lasting a minimum of 3 months. The exclusion criteria were as follows: 1) bilateral and/or pulsatile tinnitus, 2) tinnitus currently under medical treatment, 3) a previous diagnosis of neuropsychiatric or dermatological disease, and 4) the presence of an implanted metal device in the cranial region. The
Institutional Review Board of Eulji University Medical Hospital approved this study (IRB No. 2015-04-012). Written informed consent was obtained from all patients.

\section{Bifrontal tDCS+TMNMT}

tDCS was conducted using the DC-STIMULATOR PLUS (neuroConn GmbH, Ilmenau, Germany). A saline-soaked cathodal electrode and an saline-soaked anodal electrode (area $=35 \mathrm{~cm}^{2}$ ) were placed over the F3 and F4 areas, respectively, according to the 10-20 system in order to stimulate the bilateral DLPFC. Enrolled patients underwent a total of 4 sessions over a 2-week period (each Wednesday and Friday) (Fig. 1). The stimulation intensity and duration were set to $1.5 \mathrm{~mA}$ and $20 \mathrm{~min}$ (10 sec fade-in/fade-out time), respectively. During tDCS, the Tinnitus Pro app for iPad was used to provide music lacking the frequency band within 1 octave width of the patient's tinnitus frequency through a pair of headphones. For home listening, we recommended use of the same app. For patients who were not smartphone users, we created individual notched music by removing the frequency band within 1 octave width of the patient's tinnitus frequency from the patient's choice of music using the Audacity ${ }^{\circledR}$ app (available at http://audacity.sourceforge.net/). CDs of the resultant notched music were provided to each patient. All patients were instructed to listen to tailor-made notched music for a minimum of 2 hours per day.

\section{Outcome assessments}

Symptom assessments were performed upon the initial visit, directly before the first session, and 1 month after completion of the fourth session. At the initial visit, patients were screened using baseline epidemiologic characteristics, the tinnitus handicap inventory (THI), and the Beck depression inventory (BDI). In addition, the subjective severities of tinnitus loudness (LD), awareness (AW), annoyance (AN), and effect on life (EL) were assessed using a Visual Analog Scale (VAS; $0=$ no symptoms and $10=$ maximum imaginable symptoms). At the second visit, hearing status was assessed with pure tone audiometry. Mean hearing thresholds were calculated as the arithmetic mean of hearing at $0.5,1,2$, and $3 \mathrm{~Hz}$. One month after completion of the fourth session, LD, AW, AN, and EL
Fig. 1. Study design. All patients underwent 3-5 days of pre-treatment waiting, four sessions of treatment, and 28 days of washout period. During the treatment period, both bifrontal transcranial direct current stimulation (tDCS) and the tailor-made notched music training (TMNMT) were performed simultaneously.

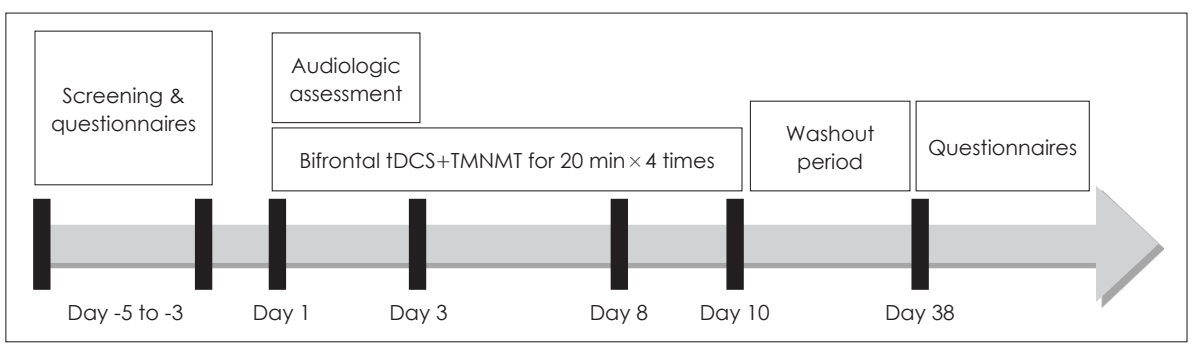


scores as well as THI and BDI scores were reassessed. The primary outcome measure was change in VAS score, and patients were deemed responders when a $50 \%$ or greater reduction was observed in the final VAS score relative to the initial VAS score. Patients were also deemed responders when a $20 \%$ or greater reduction was observed in the final THI score relative to the initial THI score. Patients who did not achieve either of these criteria were classified as non-responders. Finally, factors predicting treatment responsiveness to bifrontal tDCS+TMNMT were evaluated.

\section{Statistical analyses}

Differences between categorical variables were compared using a 2-tailed Fisher's exact test. Between group differences were assessed using the Mann-Whitney U test. Bivariate analysis was performed to evaluate the relationship between two-paired data using correlation analysis, Mann-Whitney U test and 2-tailed Fisher's exact test. A forward conditional multiple regression analysis was performed to assess predictive factors for treatment responsiveness to bifrontal tDCS+ TMNMT. All statistical analyses were performed using IBM SPSS Statistics for Macintosh, version 24.0 (IBM SPSS Statistics; IBM, Armonk, NY, USA) and p-values less than 0.05 were considered to be statistically significant.

\section{Results}

A total of 14 patients ( 9 males and 5 females) were enrolled and completed this study. The mean age was $40 \pm 13$ years (range: 20-56 years). Seven patients were affected in the right ear and 7 patients were affected in the left ear. The mean duration from onset to treatment was $28 \pm 31$ months (range: 3-84 months). The mean hearing threshold was $23 \pm 23 \mathrm{~dB}$ $\mathrm{HL}$ in the affected ear and $10 \pm 8 \mathrm{~dB} \mathrm{HL}$ in the contralateral ear. Initial VAS scores for LD, AW, AN, and EL were $4.8 \pm$ $1.9,6.7 \pm 3.1,6.0 \pm 1.8$, and $5.2 \pm 1.6$, respectively. Initial scores for the THI and BDI were $59.6 \pm 18.1$ and $11.1 \pm 9.1$, respectively.

After 4 sessions of tDCS, all VAS scores regarding tinnitus characteristics were significantly decreased $(p<0.05)$ (Fig. $2 A)$. A total of $57.1 \%(n=8)$ of patients were responders according to $\mathrm{AN}, 42.9 \%(\mathrm{n}=6)$ were responders according to AW, 35.7\% $(n=5)$ were responders according to EL, and $28.6 \%(n=4)$ were responders according to LD. On average, THI score decreased to $33.1 \pm 14.4$ after 4 sessions of tDCS $(p=0.001)$; based on THI score, $78.6 \%(\mathrm{n}=11)$ of patients were responders (Fig. 2B). No significant change was observed in BDI score $(10.5 \pm 10.7)(p=0.573)$. Three patients reported an adverse event of skin rash on the left side of the forehead; however, none of these cases required additional treatment. No other adverse events (e.g., dizziness, headache, or worsening of tinnitus) were reported.

With regard to the characteristics of responders, a bivariate analysis revealed that responders tended to have better contralateral hearing as rated by THI $(p=0.005)$ (Table 1$)$. No other clinical characteristics were found to statistically predict treatment responsiveness $(p>0.05)$. However, patients with a higher initial degree of LD tended to show improvement in LD after completion of the treatment sessions ( $p=0.054)$, and the relationships between absence of sleep disturbance and $\mathrm{AN}$ as well as EL were also marginally significant ( $p=0.063$ for both comparisons). In addition, responders tended to have
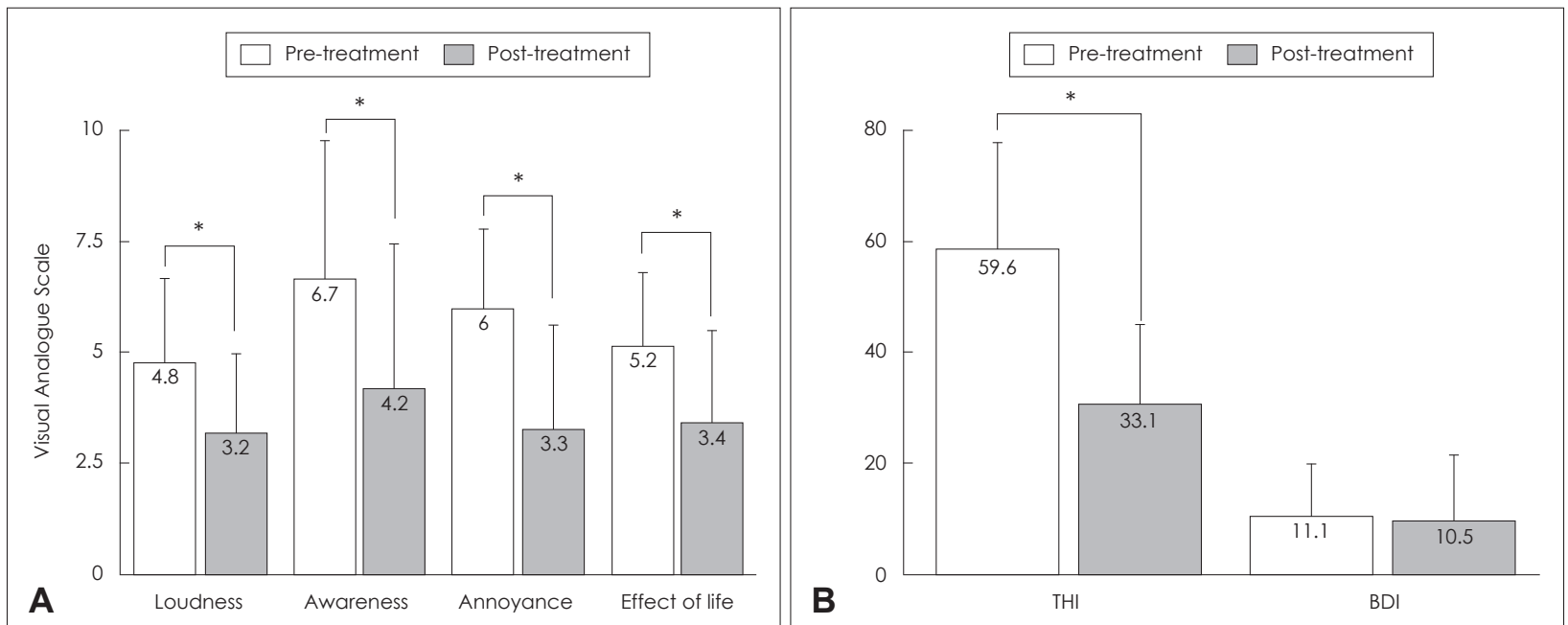

Fig. 2. Tinnitus characteristics before and after treatment. A: After treatment, tinnitus loudness, awareness, annoyance and effect on life significantly improved $\left({ }^{*} p<0.05\right)$. B: In addition, the severity of a perceived tinnitus handicap showed significant improvement $\left({ }^{*} p<0.05\right)$. However, the degree of depression remained unchanged after treatment. THI: tinnitus handicap inventory, BDI: Beck depression inventory. 
Table 1. Results of a bivariate analysis of the relationship between positive outcome and documented factors

\begin{tabular}{|c|c|c|c|c|c|}
\hline & LD & AW & AN & EL & THI \\
\hline Age & 0.304 & 0.852 & 0.662 & 0.991 & 0.368 \\
\hline Gender & 0.455 & 0.238 & 0.343 & 0.622 & 0.231 \\
\hline Side affected & 0.720 & 0.704 & 0.296 & 0.133 & 0.500 \\
\hline Duration & 0.733 & 0.414 & 0.345 & 0.951 & 0.769 \\
\hline Hearing of the affected side & 0.539 & 0.852 & 0.282 & 0.991 & 0.368 \\
\hline Hearing of the non-affected ear & 0.374 & 0.573 & 0.950 & 0.951 & $0.005^{*}$ \\
\hline Initial VAS LD & 0.054 & 0.108 & 0.414 & 1.000 & 1.000 \\
\hline Initial VAS AW & 0.188 & 0.142 & 0.414 & 0.991 & 0.225 \\
\hline Initial VAS AN & 0.945 & 0.282 & 0.181 & 0.991 & 0.885 \\
\hline Initial VAS EL & 0.945 & 0.059 & 0.414 & 0.951 & 0.885 \\
\hline Initial THI & 0.733 & 0.414 & 1.000 & 0.486 & 0.225 \\
\hline Initial BDI & 0.909 & 0.073 & 0.755 & 0.847 & 0.282 \\
\hline Headache & 0.594 & 0.471 & 0.529 & 0.343 & 0.055 \\
\hline Sleep disturbance & 0.126 & 0.343 & 0.063 & 0.063 & 0.725 \\
\hline
\end{tabular}

*The values were expressed by p-value. AW: awareness, AN: annoyance, BDI: Beck's depression inventory, EL: effect on life, LD: loudness, THI: tinnitus handicap inventory, VAS: Visual Analogue Scale

a higher initial BDI score $(p=0.073)$ and a lesser initial degree of EL ( $p=0.059)$ for AW. A forward conditional multiple regression analysis using age, gender, side affected, duration, hearing level of the both ears, initial THI and BDI, headache and sleep disturbance as covariates revealed that the absence of sleep disturbance was associated with treatment responsiveness in terms of AN ( $p=0.041, \mathrm{OR}=24.0)$. Other variables were excluded.

\section{Discussion}

tDCS has been utilized for therapeutic purposes in stroke, refractory epilepsy, chronic depression, fibromyalgia, and traumatic spinal cord injury [3]. In the present study, we found that a 2-week treatment regimen of bifrontal tDCS+TMNMT improved various aspects of tinnitus. According to THI score, $79 \%$ of the enrolled patients were responders. This result is different from that of a previous study that used TMNMT + tDCS of the LTA to treat tinnitus [10]. The effect of tDCS on tinnitus in the literature seems to vary according to study despite the use of similar methodologies; reported effects range from absent to significant in terms of tinnitus intensity and AN [3,6,10-13].

We selected the DLPFC as the target stimulation site for tDCS. The PFC is involved in memory storage and attention [11] and is associated with the integration of sensory and emotional aspects of tinnitus $[10,14]$. Several studies have reported improvements in tinnitus AN by modulating the excitability of the DLPFC with right anode-left cathode tDCS [6,12-14]. Yet, reported changes in tinnitus intensity were conflicting in some of these studies [13,14]. Further, anodal
tDCS of the LTA has been reported to decrease tinnitus intensity or to produce a transient reduction in tinnitus $[5,15]$. Consistent with previous reports, we found that only $28.6 \%$ of patients were classified as responders based on changes in tinnitus loudness, whereas changes in tinnitus AN were the basis for treatment response in $57.1 \%$ of patients. These data suggest that it may be helpful to change site of stimulation according to the most uncomfortable symptom on an individual patient basis. For example, if emotional problems are the chief complaint, a clinician might consider targeting the prefrontal cortex; however, if loudness is the most troublesome symptom, modulating LTA might be a better strategy.

Polarity is another important issue in tDCS therapy. Anodal tDCS is thought to induce excitatory effects on the underlying cerebral cortex whereas cathodal tDCS is thought to produce inhibitory effects. In addition, anodal tDCS has been reported to reduce local concentrations of GABAergic while cathodal tDCS reduces local levels of glutamate [16]. Some research has indicated that the effects of cathodal stimulation are weak, such that longer and stronger stimulation is required for cathodal versus anodal stimulation [15]. In practice, studies targeting the LTA with cathodal tDCS failed to produce significant improvements in tinnitus symptoms $[5,15]$. A study targeting the DLPFC reported that left anodal-right cathodal tDCS improved depressive symptoms of tinnitus, while right anodal-left cathodal tDCS improved anxiety related to tinnitus [14]. Conversely, a previous study of combined TMNMT and tDCS of the LTA reported no influence of polarity on the observed effects [10].

In the present study, we found that patients with better contralateral hearing were more likely to respond to combined 
TMNMT and tDCS of the DLPFC, irrespective of hearing level in the affected ear. In a previous study, patients with single-sided deafness who underwent cochlear implantation reported the improvement of tinnitus as a primary benefit, whereas patients with bilateral asymmetrical hearing loss reported the restoration of hearing as a primary benefit [17]. In a previous study with patients with unilateral sudden hearing loss, we demonstrated that better contralateral hearing was associated with new-onset acute tinnitus, and that these patients subjectively experienced louder tinnitus during auditory deprivation [18]. Taken together, these data suggest that the hearing status of the contralateral ear can be used to predict the most promising course of treatment for tinnitus. However, the role of contralateral hearing in tinnitus still requires further investigation.

Conversely, there exist various studies on the relationship between hearing in the affected ear and treatment responsiveness for tinnitus. In one study, the recovery of hearing after sudden deafness in the affected ear guaranteed the improvement of tinnitus [19]. Another report identified superior 3 -year responsiveness to modified tinnitus retraining therapy in patients with better hearing in the affected ear [20]. In a study of blast-induced chronic tinnitus, patients were more likely to select hearing aids than sound generators for longterm treatment [21]; this trend can be explained by the relationship between auditory function and tinnitus symptoms.

In our results, we also found that tinnitus AN was significantly reduced by combined TMNMT and tDCS of the DLPFC in patients that did not report sleep disturbance as a tinnitus symptom. In general, $25-77 \%$ of tinnitus patients experience sleep disturbances [22]. Furthermore, lack of sleep is a prominent feature of deteriorating tinnitus in $27 \%$ of patients [23]. In older patients, organic sleep disorders such as sleep apnea or restless leg syndrome are frequently compounded with sleep disturbance caused by tinnitus. The typical treatments for tinnitus-related sleep disturbances include short-term use of GABA receptor modulators such as zolpidem and/or cognitive behavior therapy (CBT) [24]. Since we did not allow or implement the use of medications or CBT in this study, we assumed that treatments for tinnitus-related sleep disturbances might improve treatment outcomes.

Lastly, we instructed patients to listen to music lacking the frequency band within 1 octave of the tinnitus frequency as a component of tinnitus therapy. TMNMT is thought to enhance lateral inhibition and therefore decrease compensatory, persistent neural hyperactivity in tinnitus. As DLPFC is associated with auditory memory and plays an important role in auditory processing by early inhibition of input to primary auditory cortex, simultaneously performed bifrontal DLPFC may enhance lateral inhibition by TMNMT $[11,25]$. A previous study of notch width reported that removing the frequency band within a $1 / 2$ octave of the tinnitus frequency tended be more effective than removal of the frequency band within $1 / 4$ or 1 octave, although these results were not statistically significant [26]. The application of spectral notching to environmental sounds in hearing aids has also demonstrated superiority to the use of conventional hearing aids in patients with concurrent hearing loss and tinnitus [27]. Alternatively, broadband noise sound therapy has been hypothesized to facilitate habituation to tinnitus perception according to the neurophysiologic model [28]. To our knowledge, there is no consensus regarding which treatment is more effective for tinnitus. Thus, comparative studies of TMNMT and broadband noise sound therapy are necessary in the future.

The present study has several limitations. First, we found that sleep disturbance affected treatment responsiveness significantly. For variable selection, sleep disturbance was included in multiple regression analysis, though $p$ value of a bivariate analysis was marginally significant. This might affect the outcome of this study. Second, this was a small preliminary study of 14 patients that lacked a control group for comparison. Thus, we were unable to definitively identify the major factor(s) predicting treatment responsiveness to combined TMNMT and tDCS of the DLPFC. In addition, placebo effects may have exaggerated the true effect of our therapy. Accordingly, a future larger-scale crossover study with a sufficient washout period should be conducted in order to validate and expand our findings. Moreover, differences in treatment outcome according to polarity should be addressed in a future study.

In conclusion, Combined bifrontal tDCS and TMNMT over a 2-week period yielded significant improvements in patients with chronic tinnitus, although the observed response rates varied amongst specific outcome measures. Additionally, the absence of sleep disturbances may be an important factor for predicting treatment responsiveness to combined bifrontal tDCS and TMNMT therapy.

\section{Acknowledgments}

This research was supported by Eulji University in 2015.

\section{Conflicts of interest}

The authors have no financial conflicts of interest.

\section{REFERENCES}

1) Park KH, Lee SH, Koo JW, Park HY, Lee KY, Choi YS, et al. Prevalence and associated factors of tinnitus: data from the Korean National Health and Nutrition Examination Survey 2009-2011. J Epidemiol 2014;24:417-26.

2) Forogh B, Mirshaki Z, Raissi GR, Shirazi A, Mansoori K, Ahadi T. 
Repeated sessions of transcranial direct current stimulation for treatment of chronic subjective tinnitus: a pilot randomized controlled trial. Neurol Sci 2016;37:253-9.

3) Cavalcanti K, Brasil-Neto JP, Allam N, Boechat-Barros R. A double-blind, placebo-controlled study of the effects of daily tDCS sessions targeting the dorsolateral prefrontal cortex on Tinnitus Handicap Inventory and Visual Analog Scale Scores. Brain Stimul 2015;8: 978-80.

4) Stagg CJ, Nitsche MA. Physiological basis of transcranial direct current stimulation. Neuroscientist 2011;17:37-53.

5) Garin P, Gilain C, Van Damme JP, de Fays K, Jamart J, Ossemann $\mathrm{M}$, et al. Short- and long-lasting tinnitus relief induced by transcranial direct current stimulation. J Neurol 2011;258:1940-8.

6) Song JJ, Vanneste S, Van de Heyning P, De Ridder D. Transcranial direct current stimulation in tinnitus patients: a systemic review and meta-analysis. ScientificWorldJournal 2012;2012:427941.

7) Kuo MF, Paulus W, Nitsche MA. Therapeutic effects of non-invasive brain stimulation with direct currents (tDCS) in neuropsychiatric diseases. Neuroimage 2014;85 Pt 3:948-60.

8) Vanneste S, Focquaert F, Van de Heyning P, De Ridder D. Different resting state brain activity and functional connectivity in patients who respond and not respond to bifrontal tDCS for tinnitus suppression. Exp Brain Res 2011;210:217-27.

9) Okamoto H, Stracke H, Stoll W, Pantev C. Listening to tailor-made notched music reduces tinnitus loudness and tinnitus-related auditory cortex activity. Proc Natl Acad Sci U S A 2010;107:1207-10.

10) Teismann H, Wollbrink A, Okamoto H, Schlaug G, Rudack C, Pantev C. Combining transcranial direct current stimulation and tailormade notched music training to decrease tinnitus-related distress--a pilot study. PLoS One 2014;9:e89904.

11) Pal N, Maire R, Stephan MA, Herrmann FR, Benninger DH. Transcranial direct current stimulation for the treatment of chronic tinnitus: a randomized controlled study. Brain Stimul 2015;8:1101-7.

12) Vanneste S, Plazier M, Ost J, van der Loo E, Van de Heyning P, De Ridder D. Bilateral dorsolateral prefrontal cortex modulation for tinnitus by transcranial direct current stimulation: a preliminary clinical study. Exp Brain Res 2010;202:779-85.

13) Vanneste S, Walsh V, Van De Heyning P, De Ridder D. Comparing immediate transient tinnitus suppression using tACS and tDCS: a placebo-controlled study. Exp Brain Res 2013;226:25-31.

14) Faber M, Vanneste S, Fregni F, De Ridder D. Top down prefrontal affective modulation of tinnitus with multiple sessions of tDCS of dorsolateral prefrontal cortex. Brain Stimul 2012;5:492-8.

15) Fregni F, Marcondes R, Boggio PS, Marcolin MA, Rigonatti SP,
Sanchez TG, et al. Transient tinnitus suppression induced by repetitive transcranial magnetic stimulation and transcranial direct current stimulation. Eur J Neurol 2006;13:996-1001.

16) Krause B, Márquez-Ruiz J, Cohen Kadosh R. The effect of transcranial direct current stimulation: a role for cortical excitation/inhibition balance? Front Hum Neurosci 2013;7:602.

17) Mertens G, De Bodt M, Van de Heyning P. Cochlear implantation as a long-term treatment for ipsilateral incapacitating tinnitus in subjects with unilateral hearing loss up to 10 years. Hear Res 2016; 331:1-6.

18) Lee HY, Choi MS, Chang DS, Kim AY, Cho CS. Acute-onset tinnitus is associated with contralateral hearing in sudden deafness. Audiol Neurootol 2015;20:370-5.

19) Rah YC, Park KT, Yi YJ, Seok J, Kang SI, Kim YH. Successful treatment of sudden sensorineural hearing loss assures improvement of accompanying tinnitus. Laryngoscope 2015;125:1433-7.

20) Seydel C, Haupt H, Szczepek AJ, Hartmann A, Rose M, Mazurek B. Three years later: report on the state of well-being of patients with chronic tinnitus who underwent modified tinnitus retraining therapy. Audiol Neurootol 2015;20:26-38.

21) Jalilvand H, Pourbakht A, Haghani H. Hearing aid or tinnitus masker: which one is the best treatment for blast-induced tinnitus? The results of a long-term study on 974 patients. Audiol Neurootol 2015; 20:195-201.

22) Folmer RL, Griest SE. Tinnitus and insomnia. Am J Otolaryngol 2000;21:287-93.

23) Pan T, Tyler RS, Ji H, Coelho C, Gogel SA. Differences among patients that make their tinnitus worse or better. Am J Audiol 2015;24: 469-76.

24) Møller AR, Langguth B, DeRidder D, Kleinjung T. Textbook of tinnitus. New York: Springer;2011.

25) Knight RT, Scabini D, Woods DL. Prefrontal cortex gating of auditory transmission in humans. Brain Res 1989;504:338-42.

26) Wunderlich R, Lau P, Stein A, Engell A, Wollbrink A, Rudack C, et al. Impact of spectral notch width on neurophysiological plasticity and clinical effectiveness of therailor-made notched music training. PLoS One 2015;10:e138595.

27) Strauss DJ, Corona-Strauss FI, Seidler H, Haab L, Hannemann R. Notched environmental sounds: a new hearing aid-supported tinnitus treatment evaluated in 20 patients. Clin Otolaryngol 2017;42: 172-5.

28) Kim BJ, Chung SW, Jung JY, Suh MW. Effect of different sounds on the treatment outcome of tinnitus retraining therapy. Clin Exp Otorhinolaryngol 2014;7:87-93. 